\title{
Heterogeneidade de variância residual em modelos de regressão aleatória na descrição do crescimento de codornas de corte ${ }^{1}$
}

\author{
Cristina Moreira Bonafé ${ }^{2}$, Robledo de Almeida Torres $^{3}$, Rafael Bastos Teixeira ${ }^{3}$, Felipe \\ Gomes da Silva ${ }^{2}$, Mariele Freitas Sousa ${ }^{3}$, Carla Daniela Suguimoto Leite ${ }^{3}$, Luciano Pinheiro \\ da Silva ${ }^{2}$, Giovani da Costa Caetano ${ }^{4}$

\footnotetext{
${ }^{1}$ Projeto financiado pela Fapemig.

2 Departamento de Genética e Melhoramento Animal/UFV, 36570-000, Viçosa, MG.

${ }^{3}$ Departamento de Zootecnia/UFV, 36570-000, Viçosa, MG.

${ }^{4}$ Departamento de Agronomia/UFV, 36570-000, Viçosa, MG.
}

RESUMO - Para comparar a influência da heterogeneidade da variância residual nos parâmetros genéticos estimados para a curva de crescimento de codornas de corte, foram utilizados dados provenientes de 26.835 e 27.447 observações, de 3.909 e 4.040 codornas de corte das linhagens UFV-1 e UFV-2, respectivamente. Foi avaliado o peso corporal nas duas linhagens, aos 1, 7, 14, 21, 28, 35 e 42 dias de idade, por meio de modelo animal em regressão aleatória, considerando na modelagem as variâncias residuais em classes de idades. Observou-se aumento no $\log _{e} L$, significativo pelo teste da razão de verossimilhança (LRT), com o aumento do número de classes heterogêneas. Na modelagem da variância residual, foram consideradas classes de idade: homogênea: CL1 (uma classe): 1-42 dias; e as heterogêneas: CL2 (duas classes): 1 e 7-42 dias; CL3 (três classes): 1, 7 e 14-42 dias; CL4 (quatro classes): 1, 7, 14 e 21-42 dias; CL5 (cinco classes): 1, 7, 14 e 21 e 28-42 dias; CL6 (seis classes): 1, 7, 14, 21, 28 e 35-42 dias; CL7 (sete classes): 1, 7, 14, 21, 28, 35 e 42 dias de idade. O modelo que considerou homogeneidade de variância residual mostrou-se inadequado. As estimativas de variâncias, herdabilidades e correlações foram influenciadas pela modelagem da variância residual. A utilização de heterogeneidade de variância residual (CL7) para modelar as variâncias associadas à curva de crescimento das codornas de corte é necessária.

Palavras-chave: Coturnix coturnix, curva de crescimento, herdabilidade, modelo animal, parâmetros genéticos

\section{Heterogeneity of residual variance in random regression models in the description of meat quail growth}

\begin{abstract}
In order to compare the residual heterogeneity variance influence on the genetic parameters estimated for the growth curve of meat quail, data from 26835 and 27447 observations, from 3909 and 4040 meat quails of the UFV-1 and UFV-2 strains, respectively, were used. Body weight was evaluated, for the two strains, at 1, 7, 14, 21, 28, 35 and 42 days of age, through an animal model at random regression, considering residual variances in the age classes. $\log _{e} \mathrm{~L}$ showed increase, significant by the likelihood ratio test (LRT), with an increase in the number of heterogeneous classes. In the modeling, the following residual variances were considered in the age classes of: homogeneous: CL1 (one class): 1-42 days; and heterogeneous: CL2 (two classes): 1 and 7-42 days; CL3 (three classes): 1, 7 and 14-42 days; CL4 (four classes): 1, 7, 14 and 21-42 days; CL5 (five classes): 1, 7, 14, 21 and 28-42 days; CL6 (six classes): 1, 7, 14, 21, 28 and 35-42 days; CL7 (seven classes): 1, 7, 14, 21, 28, 35 and 42 days of age. The model that considered residual variance homogeneity showed unsuitable. Variance estimates, heritability and correlations were influenced by the modeling of the residual variance. The use of residual variance heterogeneity (CL7) to model the variances associated with the growth curve of meat quail is necessary.
\end{abstract}

Key Words: animal model, Coturnix coturnix, genetic parameter, growth curve, heritability

\section{Introdução}

No melhoramento genético, os modelos de regressão aleatória têm sido reconhecidos como mais apropriados para a análise de dados longitudinais, pois a utilização de metodologias que possam estimar os parâmetros genéticos de forma mais acurada pode contribuir para o aumento dos ganhos genéticos obtidos com a seleção dos animais.

Esses modelos permitem a análise de registros repetidos de características que mudam gradualmente ao longo do tempo e não requerem pressuposições quanto à constância das variâncias e correlações (Meyer, 2000) e têm sido 
utilizados no melhoramento animal para modelar registros diários de produção de leite durante a lactação (El Faro \& Albuquerque, 2003; Breda et al., 2006) para modelar o crescimento corporal de animais (Sakaguti et al., 2003; Fischer et al., 2004; Sarmento et al., 2006), assim como em estudos de normas de reação, que avaliam a sensibilidade do genótipo às variações de ambiente (Meyer \& Hill, 1997; Kolmodin et al., 2002).

Os primeiros estudos utilizando modelos de regressão aleatória (Jamrozik et al., 1997; Jamrozik \& Schaeffer, 1997) desconsideravam estrutura heterogênea de variâncias residuais, o que proporcionava superestimações das variâncias genéticas aditivas. A modelagem considerando heterogeneidade de variâncias residuais para cada idade, por sua vez, pode melhorar a partição da variação total, mas também proporciona aumento dos parâmetros a serem estimados no processo de maximização da função de verossimilhança (El Faro \& Albuquerque, 2003), o que pode dificultar a utilização dos modelos de regressão aleatória em avaliações genéticas com grande volume de dados. Neste sentido, busca-se a utilização de modelos mais parcimoniosos, sem perda da qualidade de ajuste dos efeitos aleatórios considerados.

Diversos estudos propõem a modelagem das variâncias residuais por meio de classes heterogêneas ou funções de variâncias residuais, empregando polinômios ortogonais de Legendre ou ordinários em ovinos de corte (Lewis \& Brotherstone, 2002; Sarmento et al., 2010), em bovinos de leite (Torres, 2000; El Faro \& Albuquerque, 2003), em bovinos de corte (Meyer, 2000) e em caprinos (Sousa et al., 2008).

Os poucos trabalhos com codornas de corte encontrados na literatura consultada (Akbas et al., 2004; Dionello et al., 2006) assumiram homogeneidade de variância residual.

Desta forma, objetivou-se com este trabalho comparar a influência da heterogeneidade da variância residual na estimação dos componentes de variância e parâmetros genéticos na descrição da curva de crescimento de codornas de corte utilizando Modelos de Regressão Aleatória.

\section{Material e Métodos}

Os dados utilizados neste estudo são provenientes de duas linhagens experimentais de codornas de corte, pertencentes ao Programa de Melhoramento Genético de Aves da Universidade Federal de Viçosa. Foram utilizados 26.835 e 27.447 registros de pesos, mensurados ao nascimento e aos $7,14,21,28,35$ e 42 dias de idade, provenientes de 3.909 e 4.040 codornas de corte, com 6.742 e 7.381 animais na matriz de parentesco, das linhagens UFV-1 e UFV-2, respectivamente.
Os pesos corporais foram analisados por meio de regressão aleatória utilizando o modelo animal. As regressões fixas e aleatórias foram representadas por funções contínuas, cujas idades foram descritas em termos de polinômios ortogonais de Legendre, de ordem 3 (quadrático).

A generalização dos modelos com diferentes estruturas de variâncias residuais pode ser representada como segue:

$y_{i j}=\sum_{m=0}^{k_{\beta}-1} \beta_{m} \phi_{m}+\sum_{m=0}^{k_{a}-1} \alpha_{i m} \phi_{m}+\sum_{m=0}^{k_{c}-1} \rho_{i m} \phi_{m}+\varepsilon_{i j}$

em que: $y_{i j}=$ peso no dia $j$, da codorna $i ; F=$ efeitos fixos incluídos no modelo (grupo de contemporâneos incluindo geração, eclosão, sexo e idade, gerando 126 classes); $\beta_{m}=$ coeficiente de regressão fixo $m$ do peso sobre o polinômio de Legendre, $\operatorname{com} k_{\beta}=3$ (função quadrática), para modelar a curva média de crescimento da população; $\alpha_{i m}$ e $\rho_{i m}=$ coeficientes de regressão genético aditivo direto e de ambiente permanente do animal, respectivamente, para a codorna $i ; k_{a}=3$ e $k_{c}=3$ são as ordens dos polinômios a serem ajustados; $\phi_{m}=$ função polinomial de legendre $m$ da idade padronizada $(-1<$ idade $<1)$; e $\varepsilon_{i j}=$ efeito aleatório residual.

Na modelagem da variância residual, foram consideradas classes de idade: homogênea: CL1 (uma classe): 1-42 dias; e as heterogêneas: CL2 (duas classes): 1 e 7-42 dias; CL3 (três classes): 1, 7 e 14-42 dias; CL4 (quatro classes): 1, 7, 14 e 21 42 dias; CL5 (cinco classes): 1, 7,14 e 21 e 28-42 dias; CL6 (seis classes): 1, 7, 14, 21, 28 e 35-42 dias; CL7 (sete classes): 1, 7, 14, 21, 28, 35 e 42 dias de idade, com o total de sete diferentes modelos para serem utilizados nas análises dos dados.

Na forma matricial, o modelo anterior, com suas respectivas pressuposições, pode ser escrito como:

$y=X b+Z_{1} a+Z_{2} c+e$

$E\left[\begin{array}{l}y \\ a \\ c \\ e\end{array}\right]=\left[\begin{array}{c}X b \\ 0 \\ 0 \\ 0\end{array}\right] \quad$ e $\quad \begin{gathered}\operatorname{Var}(a)=K_{a} \otimes A \\ \operatorname{Var}(e)=R\end{gathered}$

em que: $y=$ vetor de $\mathrm{N}$ observações referentes a $N_{d}$ animais, em que $N_{d}$ denota o número total de animais na matriz dos numeradores do coeficiente de parentesco entre os indivíduos (6.742 para UFV-1 e 7.381 para UFV-2); $b=$ vetor que contém os efeitos fixos e os coeficientes $b_{m}$ da regressão fixa; $a=$ vetor $k_{a} \times N_{d}$ de coeficientes de regressão aleatória genéticos aditivos diretos; $c=$ vetor $k_{c} \times N_{d}$ de coeficientes de regressão aleatória ambiente permanente de animal; $e=$ vetor de erros aleatórios; $X, Z_{1}$ e $Z_{2}=$ matrizes de incidência dos coeficientes de regressão fixos e efeitos fixos (grupos contemporâneos), coeficientes de regressão aleatória genético aditivo direto e ambiente 
permanente de animal, respectivamente. $K_{a}$ e $K_{c}=$ matrizes de covariâncias entre os coeficientes de regressão aleatórios genético aditivo direto e ambiente permanente de animal, respectivamente; $A=$ matriz dos numeradores do coeficiente de parentesco entre os indivíduos; $I_{N d}=$ matriz identidade de dimensão $N_{d} ; \otimes=$ operador produto direto; $R=$ matriz diagonal de variâncias residuais com elementos que dependem do número de classes ajustadas, ou seja, $\left[\sigma_{j}^{2}\right]$.

As covariâncias entre os coeficientes de regressão aleatória genéticos aditivos direto e ambiente permanente de animal, conforme o modelo ajustado, foram estimadas pelo método da máxima verossimilhança restrita (REML), usando o programa DXMRR do software DFREML (Meyer, 1998).

A comparação entre os modelos foi feita pelo Critério de Informação de Akaike (AIC), Critério de Informação Bayesiano de Schwarz (BIC), Logaritmo da função de verossimilhança $\left(\log _{e} L\right)$ e teste da razão de verossimilhança (LRT), a 1\% de probabilidade, para testar diferenças entre os modelos estudados.

Os critérios AIC e BIC foram obtidos por: $A I C=-2 \log _{e} L+2 p$ e $B I C=-2 \log _{e} L+2 p \log _{e}(N-r)$, em que p refere-se ao número de parâmetros do modelo, $\mathrm{N}$ é o número total de observações e $r$ é o posto da matriz de incidência para os efeitos fixos. Menores valores de AIC e BIC e maiores valores de $\log _{\mathrm{e}} \mathrm{L}$ indicam modelos mais adequados. A estatística LRT foi obtida pela seguinte expressão: $\mathrm{LRT}_{\mathrm{ij}}=2 \log _{\mathrm{e}} \mathrm{L}_{\mathrm{i}}-2 \log _{\mathrm{e}} \mathrm{L}_{\mathrm{j}}$, em que $\log _{\mathrm{e}} \mathrm{L}_{\mathrm{i}}$ é o máximo da função de verossimilhança para o modelo completo i e $\log _{e} L_{j}$ o máximo da função de verossimilhança para o modelo reduzido j. A estimativa LRT foi comparada com o valor do qui-quadrado tabelado, com d graus de liberdade e nível de significância de $1 \%$, sendo d a diferença entre o número de parâmetros estimados pelos modelos completo e reduzido (aninhados). Para comparar os resultados obtidos pelos modelos de regressão aleatória, foram realizadas, no mesmo conjunto de dados, análises unicaracterísticas por meio de modelo animal, que incluiu o mesmo efeito fixo (grupo contemporâneo) e o efeito aleatório genético direto. Os componentes de covariância e os parâmetros genéticos foram estimados pelo programa MTDFREML, desenvolvido por Boldman et al. (1995).

As mudanças ocorridas nos componentes de variância e os parâmetros genéticos estimados ao longo da curva de crescimento foram avaliados em função do ajuste da variância residual.

\section{Resultados e Discussão}

No resumo das análises (Tabela 1), considerando-se inicialmente a modelagem do resíduo, por meio de classes de variância, observaram-se reduções $(\mathrm{p}<0,01)$ no $\log _{\mathrm{e}} \mathrm{L}$ à medida que aumentou o número de classes de variância residual até sete (CL7). De acordo com todos os critérios utilizados para avaliar a qualidade de ajuste, o modelo que considerou homogeneidade de variância residual (CL1) foi o menos adequado. A diferença obtida entre os modelos CL1 e CL2, pelo teste LRT, foi significativa ( $<<0,01$ ), o que indica que o CL2 pode ser utilizado no ajuste da variância residual. No entanto, na comparação dos modelos que utilizaram classes de variâncias por meio dos critérios AIC e BIC, o melhor resultado, quanto ao ajuste da variância residual, foi observado com a utilização do modelo CL7.

Sarmento et al. (2010), trabalhando com a curva de crescimento de ovinos, consideraram heterogeneidade entre classes de idade. Esses autores verificaram que o modelo

Tabela 1 - Teste para comparação dos modelos

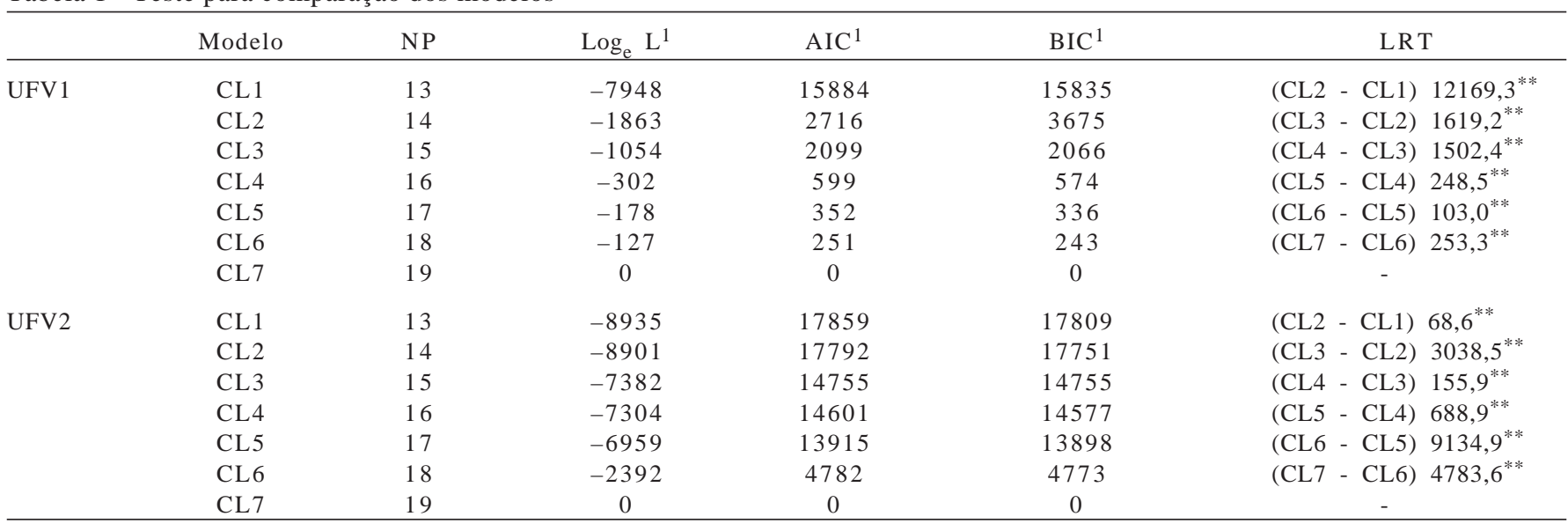

** Significativo a $1 \%$ de probabilidade.

${ }^{1}$ Valores expressos como desvio do melhor valor.

CL = classes de idades; NP: número de parâmetros; $\log _{e} \mathrm{~L}=\log$ aritimo da função de verossimilhança; AIC = critério de informação de Akaike; BIC = critério de informação Bayesiano de Schwarz; LTR = teste da razão de verossimilhança. 
considerando homogeneidade de variância residual mostrou-se inadequado. O modelo contendo sete classes proporcionou melhor ajuste, embora um modelo mais parcimonioso, com cinco classes, pudesse ser utilizado sem perdas na qualidade de ajuste da variância nos dados.
O fato de assumir homogeneidade de variância residual ao longo da curva de crescimento resulta em distorções consideráveis na partição da variância total, uma vez que houve grande diferença nas estimativas de variâncias residuais (Figura 1).
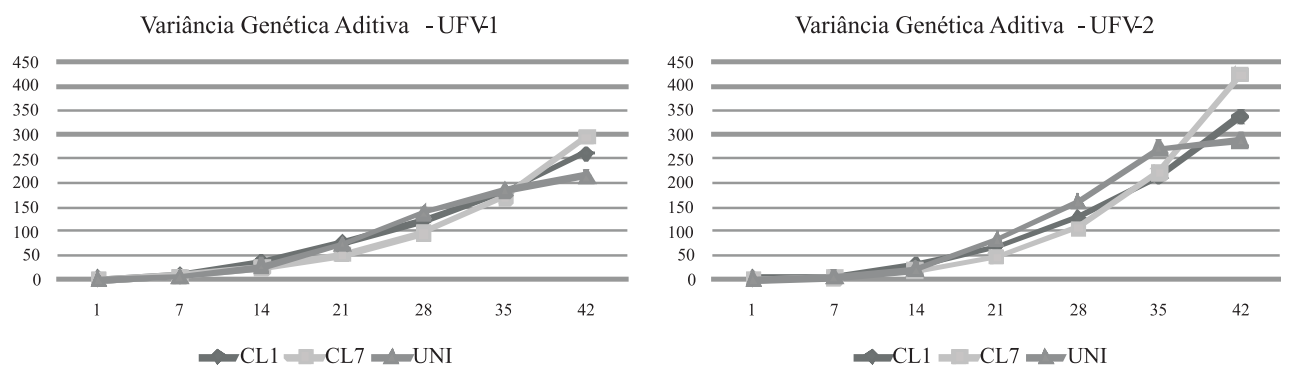

Variância de Ambiente Permanente - UFV-1

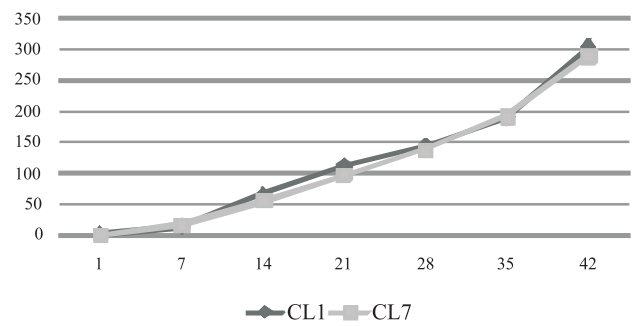

Variância Fenotípica - UFV-1

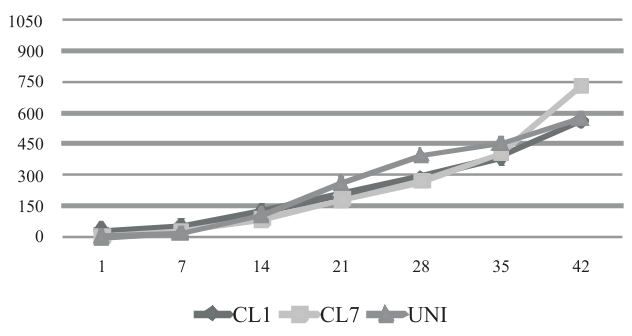

Variância de Ambiente Permanente - UFV2

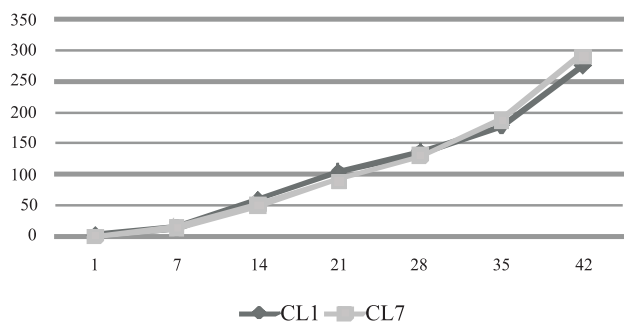

Variância Fenotípica - UFV2

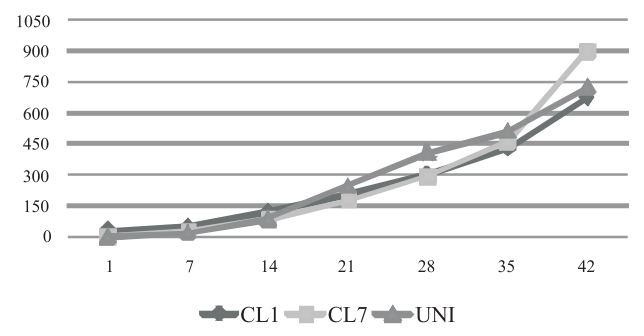

Variância Residual - UFV-1

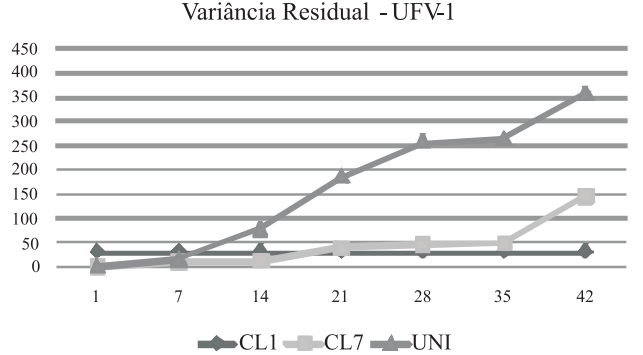

Variância Residual - UFV2

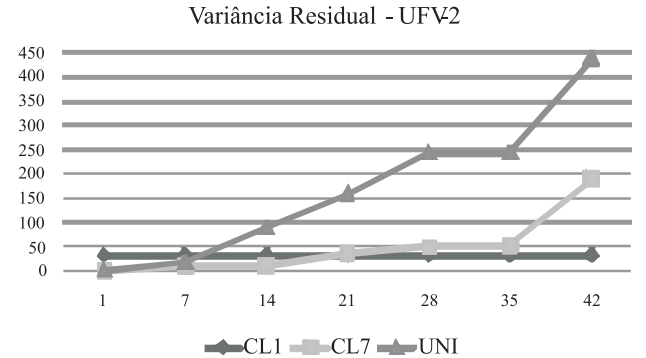

Herdabilidade - UFV1
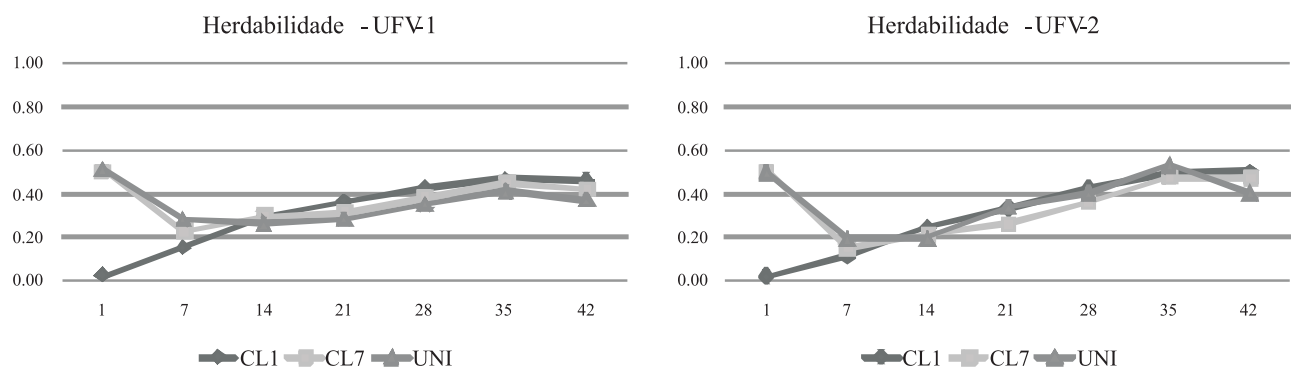

Figura 1 - Estimativas de variâncias e herdabilidades para os modelos com homogeneidade de variância residual (CL1), heterogeneidade de variância residual com 7 classes (CL7) e análises unicaracterísticas (UNI). 
A variância genética aditiva direta também foi influenciada pela modelagem da variância residual, com mudanças maiores no final da curva de crescimento, quando se considerou heterogeneidade de variância.

As estimativas de variância atribuídas ao ambiente permanente foram similares entre os modelos que consideram homogeneidade e heterogeneidade de variância residual. A variância de ambiente permanente aumentou com a idade para os dois modelos avaliados, à semelhança dos resultados obtidos por Akbas et al. (2004), com homogeneidade de variância residual, cujas estimativas de variância de ambiente permanente variaram de 60,63 a 835,90 .
As estimativas de herdabilidades diretas ao longo da curva de crescimento diferiram em função do ajuste da variância residual. No início da curva, as herdabilidades estimadas quando se admitem a variância residual como heterogênea e os resultados das análises unicaracterísticas foram maiores que as estimadas ao se considerar a variância residual homogênea.

As herdabilidades estimadas foram crescentes do nascimento até 35 dias de idade no modelo CL1, e de 7 a 35 dias nos modelos CL7 e UNI, com ligeiro decréscimo de 35 a 42 dias de idade.

Akbas et al. (2004), considerando variância residual homogênea, encontraram estimativas de herdabilidade

Tabela 2 - Estimativas das correlações genéticas entre os pesos nas idades

\begin{tabular}{|c|c|c|c|c|c|c|c|c|}
\hline & & & & & UFV-1 & & & \\
\hline & & & & & CL1 & & & \\
\hline & Idade & 1 & 7 & 14 & 21 & 28 & 35 & 42 \\
\hline & 1 & & $-1,00$ & $-0,99$ & $-0,95$ & $-0,89$ & $-0,78$ & $-0,64$ \\
\hline & 7 & 0,46 & & 0,99 & 0,96 & 0,90 & 0,80 & 0,66 \\
\hline & 14 & 0,41 & 0,98 & & 0,99 & 0,95 & 0,87 & 0,75 \\
\hline CL 7 & 21 & 0,41 & 0,92 & 0,98 & & 0,99 & 0,93 & 0,84 \\
\hline & 28 & 0,42 & 0,82 & 0,91 & 0,97 & & 0,98 & 0,92 \\
\hline & 35 & 0,41 & 0,68 & 0,80 & 0,91 & 0,98 & & 0,98 \\
\hline & 42 & 0,39 & 0,54 & 0,67 & 0,81 & 0,92 & 0,98 & \\
\hline & & & & & UFV-2 & & & \\
\hline & & & & & CL1 & & & \\
\hline & Idade & 1 & 7 & 14 & 21 & 28 & 35 & 42 \\
\hline & 1 & & $-0,97$ & $-1,00$ & $-0,99$ & $-0,95$ & $-0,89$ & $-0,80$ \\
\hline & 7 & 0,50 & & 0,98 & 0,93 & 0,85 & 0,74 & 0,62 \\
\hline & 14 & 0,42 & 0,96 & & 0,98 & 0,94 & 0,87 & 0,77 \\
\hline CL 7 & 21 & 0,40 & 0,86 & 0,97 & & 0,98 & 0,94 & 0,87 \\
\hline & 28 & 0,38 & 0,74 & 0,89 & 0,98 & & 0,99 & 0,94 \\
\hline & 35 & 0,36 & 0,62 & 0,80 & 0,93 & 0,99 & & 0,99 \\
\hline & 42 & 0,33 & 0,52 & 0,72 & 0,87 & 0,96 & 0,99 & \\
\hline
\end{tabular}

CL1 = homogeneidade de variância residual (acima da diagonal); CL7 = heterogeneidade de variância residual (abaixo da diagonal).

Tabela 3 - Estimativas das correlações de ambiente permanente entre as idades

\begin{tabular}{|c|c|c|c|c|c|c|c|c|}
\hline & \multirow[b]{3}{*}{ Idade } & \multicolumn{7}{|c|}{ UFV-1 } \\
\hline & & \multicolumn{7}{|c|}{ CL1 } \\
\hline & & 1 & 7 & 14 & 21 & 28 & 35 & 42 \\
\hline & 1 & & $-1,00$ & $-0,98$ & $-0,93$ & $-0,81$ & $-0,55$ & $-0,20$ \\
\hline & 7 & 0,23 & & 0,99 & 0,96 & 0,85 & 0,62 & 0,28 \\
\hline & 14 & 0,15 & 0,99 & & 0,98 & 0,91 & 0,71 & 0,39 \\
\hline \multirow[t]{10}{*}{ CL7 } & 21 & 0,12 & 0,94 & 0,98 & & 0,97 & 0,82 & 0,55 \\
\hline & 28 & 0,09 & 0,84 & 0,91 & 0,97 & & 0,94 & 0,74 \\
\hline & 35 & 0,05 & 0,66 & 0,76 & 0,87 & 0,96 & & 0,93 \\
\hline & 42 & 0,02 & 0,42 & 0,55 & 0,69 & 0,84 & 0,96 & \\
\hline & & & & & UFV-2 & & & \\
\hline & Idade & & & & CL 1 & & & \\
\hline & & 1 & 7 & 14 & 21 & 28 & 35 & 42 \\
\hline & 1 & & $-0,99$ & $-0,96$ & $-0,90$ & $-0,75$ & $-0,46$ & $-0,08$ \\
\hline & 7 & 0,31 & & 0,99 & 0,96 & 0,84 & 0,59 & 0,24 \\
\hline & 14 & 0,24 & 0,99 & & 0,98 & 0,90 & 0,68 & 0,35 \\
\hline \multirow[t]{4}{*}{ CL7 } & 21 & 0,21 & 0,95 & 0,98 & & 0,96 & 0,80 & 0,51 \\
\hline & 28 & 0,17 & 0,85 & 0,92 & 0,97 & & 0,93 & 0,72 \\
\hline & 35 & 0,12 & 0,67 & 0,76 & 0,87 & 0,96 & & 0,92 \\
\hline & 42 & 0,06 & 0,42 & 0,54 & 0,68 & 0,83 & 0,96 & \\
\hline
\end{tabular}

CL1 = homogeneidade de variância residual (acima da diagonal); CL7 = heterogeneidade de variância residual (abaixo da diagonal). 
baixa $(0,007)$ para o peso ao nascer, semelhante a encontrada no modelo CL1, já para as demais idades, as estimativas encontradas foram ligeiramente maiores.

As correlações entre os pesos nas várias idades, para os dois modelos estudados, à exceção das correlações entre peso ao nascer e os demais pesos, foram altas e positivas (Tabelas 2 e 3), à semelhança dos resultados encontrados por Akbas et al. (2004), com homogeneidade de variância residual. No modelo com homogeneidade de variância residual, as correlações relacionadas com o peso ao nascimento foram negativas, entretanto de maior magnitude em relação à do modelo que considerou heterogeneidade de variância residual.

Wenceslau et al. (2007) avaliaram duas linhagens de codornas de corte no período de crescimento, com homogeneidade de variância, e também encontraram correlações genéticas positivas, de média a alta magnitude, entre os pesos corporais de 21 a 42 dias de idade.

As correlações de ambiente permanente estimadas entre os pesos tenderam a ser maiores entre idades mais próximas, à exceção daqueles entre o peso ao nascer (1 dia) e os pesos nas demais idades. As correlações entre o peso ao nascer e os demais pesos (Tabela 3) apresentaram valores baixos pelo modelo CL7 e negativos pelo modelo CL1.

\section{Conclusões}

As estimavas de variâncias, herdabilidades e correlações são influenciadas pela modelagem da variância residual, tornando necessária a utilização de heterogeneidade de variância residual para modelar as variâncias residuais associadas à curva de crescimento das codornas de corte.

\section{Agradecimentos}

À FAPEMIG, pelo financiamento do projeto, à CAPES e ao $\mathrm{CNPq}$, pelas bolsas de estudo concedidas.

\section{Referências}

AKBAS, Y.; TAKMA, Ç.; YAYLAK, E. Genetic parameters for quail body weights using a random regression model. South African Journal of Animal Science, v.34, n.2, p.104-109, 2004

BOLDMAN, K.G.; KRIESE, L.A.; VAN VLECK, L.D. et al. A manual for use of MTDFREML. A set of programs to obtain estimates of variance and covariance [DRAFT]. Lincoln: USDA/ ARS. 1995. 120p.

BREDA, F.C.; ALBUQUERQUE, L.G.; YAMAKI, M. et al. Estimação de parâmetros genéticos para produção de leite de cabras da raça Alpina. Revista Brasileira de Zootecnia, v.35, p.396-404, 2006.
DIONELLO, N.J.L.; CORRÊA, G.S.S.; SILVA, M.A. et al. Efeitos maternos e permanentes na avaliação genética de linhagens de codornas de corte utilizando modelos de regressão aleatória. REUNIÃO ANUAL DA SOCIEDADE BRASILEIRA DE ZOOTECniA, 43., 2006, João Pessoa. Anais... João Pessoa: SBZ, 2006. (CD-ROM)

EL FARO, L.; ALBUQUERQUE, L.G. Utilização de modelos de regressão aleatória para produção de leite no dia de controle, com diferentes estruturas de variâncias residuais. Revista Brasileira de Zootecnia, v.32, n.5, p.1104-1113. 2003.

FISCHER, T.M.; VAN DER WERF, J.H.J.; BANKS, R.G. et al. Description of lamb growth using random regression on $\mathrm{fi}$ eld data. Livestock Production Science, v.89, p.175-185, 2004.

JAMROZIK, J.; KISTEMAKER, G.J.; DEKKERS, J.C.M. et al. Comparison of possible covariates for use in a random regression model for analyses of test day yields. Journal of Dairy Science, v.80, p.2550-2556, 1997.

JAMROZIK, J.; SCHAEFFER, L.R. Estimates of genetic parameters for a test day model with random regression for yield traits of first lactation Holsteins. Journal of Dairy Science, v.80, p.762-770, 1997.

KOLMODIN, R.; STRANDBERG, E.; MADSEN, P. et al. Genotype by environment interaction in Nordic dairy cattle studied using reaction norms. Acta Agriculturae Scandinavica; Section A - Animal Sciences, v.52, p.11-24, 2002.

LEWIS, R.M.; BROTHERSTONE, S. A genetic evaluation of growth in sheep using random regression techniques. Animal Science, v.74, p.63-70, 2002.

MEYER, K.; HILL, W.G. Estimation of genetic and phenotypic covariance functions for longitudinal or "repeated" records by restricted maximum likelihood. Livestock Production Science, v.47, p.185-200, 1997.

MEYER, K. "DXMRR” - A program to estimate covariance functions for longitudinal data by REML. WORLD CONGRESS OF GENETICS APPLIED TO LIVESTOCK PRODUCTION, 6., 1998, Armidale. Proceedings... Armidale: University of New England, 1998. (CD-ROM).

MEYER, K. Random regression to model phenotypic variation in monthly weights of Australian beef cows. Livestock Production Science, v.65, p.19-38, 2000.

SAKAGUTI, E.S.; SILVA, M.A.; QUAAS, R.L. Avaliação do crescimento de bovinos jovens da raça Tabapuã, por meio de análise de funções de covariância. Revista Brasileira de Zootecnia, v.32, p.864-874, 2003.

SARMENTO, J.L.R.; TORRES, R.A.; PEREIRA, C.S. et al Avaliação genética de características de crescimento de ovinos Santa Inês utilizando modelos de regressão aleatória. Arquivo Brasileiro de Medicina Veterinária e Zootecnia, v.58, p.68-77, 2006.

SARMENTO, J.L.R; TORRES, R.A.; LÔBO, R.N.B. et al, Modelos de regressão aleatória na avaliação genética do crescimento de ovinos da raça Santa Inês. Revista Brasileira de Zootecnia, v.39, n.8, p.1723-1732, 2010.

SOUSA, J.E.R.; SILVA, M.A.; SARMENTO, J.L.R. et al. Homogeneidade e heterogeneidade de variância residual em modelos de regressão aleatória sobre o crescimento de caprinos Anglo-Nubianos. Pesquisa Agropecuária Brasileira, v.43, n.12, p.1725-1732, 2008.

TORRES, R.A.; BERGMANN, J.A.G.; COSTA, C.N. et al, Heterogeneidade de variância e avaliação genética de bovinos da raça Holandesa no Brasil. Revista Brasileira de Zootecnia, v.29, n.4, p.1050-1059, 2000.

WENCESLAU, R.R.; CORRÊA, G.S.S.; CORRÊA, A.B. et al. Correlação genética e herdabilidade de pesos vivos de codornas de corte avaliados no período de crescimento. SIMPÓSIO INTERNACIONAL DE COTURNICULTURA, 2007, Lavras. Anais... Lavras: UFLA, 2007. 